\title{
Neuroinflammation and neurohormesis in the pathogenesis of Alzheimer's disease and Alzheimer-linked pathologies: modulation by nutritional mushrooms
}

\author{
Angela Trovato Salinaro ${ }^{1 \dagger}$, Manuela Pennisi ${ }^{1,4+}$, Rosanna Di Paola ${ }^{2 \dagger}$, Maria Scuto ${ }^{1}$, Rosalia Crupi ${ }^{2}$, \\ Maria Teresa Cambria', Maria Laura Ontario ${ }^{1}$, Mario Tomasello ${ }^{1}$, Maurizio Uva ${ }^{3}$, Luigi Maiolino', \\ Edward J. Calabrese ${ }^{5}$, Salvatore Cuzzocrea ${ }^{2}$ and Vittorio Calabrese ${ }^{1 * \dagger}$
}

\begin{abstract}
Human life develops and expands not only in time and space, but also in the retrograde permanent recollection and interweaving of memories. Therefore, individual human identity depends fully on a proper access to the autobiographical memory. Such access is hindered or lost under pathological conditions such as Alzheimer's disease, including recently associated oxidant pathologies, such as ocular neural degeneration occurring in glaucoma or neurosensorial degeneration occurring in Menière's disease. Oxidative stress and altered antioxidant systems have been suggested to play a role in the aetiology of major neurodegenerative disorders, and altered expression of genes sensing oxidative stress, as well as decreased cellular stress response mechanisms could synergistically contribute to the course of these oxidant disorders. Thus, the theory that low levels of stress can produce protective responses against the pathogenic processes is a frontier area of neurobiological research focal to understanding and developing therapeutic approaches to neurodegenerative disorders. Herein, we discuss cellular mechanisms underlying AD neuroinflammatory pathogenesis that are contributory to Alzheimer's disease. We describe endogenous cellular defence mechanism modulation and neurohormesis as a potentially innovative approach to therapeutics for AD and other neurodegenerative conditions that are associated with mitochondrial dysfunction and neuroinflammation. Particularly, we consider the emerging role of the inflammasome as an important component of the neuroprotective network, as well as the importance of Coriolus and Hericium nutritional mushrooms in redox stress responsive mechanisms and neuroprotection.
\end{abstract}

Keywords: Oxidative stress, Neurodegenerative disorders, Neurohormesis, Mushrooms

\section{Background}

Alzheimer's disease (AD) is a neurodegenerative progressive disorder affecting more than 15 million people worldwide and represents the most current cause of dementia in the elderly, accounting for $50-60 \%$ of all cases in Western world [1].

The pathological signs of $\mathrm{AD}$ are amyloid plaques containing amyloid- $\beta(A \beta)$ peptide derived from transamyloid precursor protein and neurofibrillary tangles

\footnotetext{
*Correspondence: calabres@unict.it

${ }^{\dagger}$ Equal contributors

${ }^{1}$ Department of Biomedical and Biotechnological Sciences, School of Medicine, University of Catania, Via Santa Sofia 97, 95123 Catania, Italy Full list of author information is available at the end of the article
}

constituted by hyper phosphorylated tau protein in medial temporal lobe structures and cortical areas of the brain along with neuronal death and synapse loss [2]. It has been demonstrated that inflammation cascade is linked to neurodegenerative diseases, particularly, Alzheimer's disease (AD) [3, 4]. In order to resist to different injuries, brain cells have developed networks of responses that detect and control different forms of stress $[5,6]$. These are mainly proteins, including heat shock proteins (Hsps), lipoxin A4 (LXA4), thioredoxin (Trx) and sirtuins, controlled by vitagenes, a redoxdependent complex of genes [7]. LXA4 is an endogenous eicosanoid, produced by arachidonic acid metabolism, endowed with anti-inflammatory properties in different 
inflammatory syndromes, such as periodontitis, nephritis, arthritis, inflammatory bowel disease. LXA4 blocks the productions of pro-inflammatory mediators including free radical oxygen and nitrogen reactive intermediates (ROS/RNS), and acts as an endogenous "braking signal" in the inflammatory process. It is generally acknowledged as "signal stop" of inflammation [8]. The discovery of agents capable of increasing LXA4 levels and subsequently $A \beta$ uptake by phagocytic cells is increasingly being recognized as a potential therapeutic target for $\mathrm{AD}$ treatment. Previous studies have demonstrated that mushrooms significantly upregulate LXA4 in the brain. Mushrooms have been used in traditional medicine, for many years [9], and a long list of therapeutic properties exists that have been associated with mushroom extracts, including antitumor, immunomodulatory, antioxidant, antiviral, antibacterial, and hepatoprotective effects. Mushrooms are a rich font of polysaccharides, and many of them have been shown to stimulate host immune responses. Among the most powerful known immunostimulators, $\beta$-glucans derived from mushrooms stimulate immune cells and cytokine responses $[8,10]$.

Taken into account the fact that Alzheimer's disease is characterized by neurodegeneration associated with neuroinflammation, in our recent study we have shown that Coriolus versicolor and Hericium erinaceus biomass preparations have neuroprotective effects and act by modulating the inflammatory process associated with the pathology of $\mathrm{AD}$, as well as regulating brain cellular stress response mechanisms [4].

There is a growing body of evidence demonstrating a link between Alzheimer's disease and glaucoma. Notably, amyloid deposits, constituted of amyloid beta $(\mathrm{A} \beta)$ aggregates, a characteristic feature of several neurodegenerative diseases, such as Alzheimer's, mild cognitive impairment and Parkinson's disease (PD), have been recently implicated in the pathogenesis of retinal damage, of age-related macular degeneration and glaucoma. Glaucoma is a progressive optic neuropathy characterized by gradual degeneration of neuronal tissue due to retinal ganglion cell loss, associated to visual field loss over time resulting in irreversible blindness $[11,12]$. It is a leading cause of irreversible blindness estimated to affect 79.6 million people worldwide by 2020 . Accumulation of $A \beta$ characterizes glaucoma as a protein misfolding disease, suggesting a pathogenic role for oxidative stress in the pathogenesis of retinal degenerative damage associated to this ocular pathology. In particular, factors such as tissue hypoxia and disturbed protein metabolism have been identified to interact in a vicious cycle underlying the oxidative stress-driven pathogenesis of glaucoma, ultimately leading to apoptotic retina ganglion cell death [13]. Research studies have demonstrated that retinal ganglion cell (RGC) damage in glaucoma is not limited to the primary insulted neurons, but also involves neighbouring neurons. In view of these considerations glaucoma can be viewed as a neurodegenerative disease which, similarly to other neurodegenerative pathologies, i.e., Alzheimer's and Parkinson's disease, where irreversible functional deficit ensues as consequence of neuronal dysfunction and death. Interestingly, recent evidence from our laboratory have demonstrated higher levels of vitagenes Heat Shock Protein 72 (Hsp72) and Heme oxygenase (HO-1) in the blood of patients with glaucoma than in controls [14]. These changes were associated with an increased expression of Trx and sirtuin 1 in the same experimental group. Similar results have been found in another oxidant disorder impacted by a progressive degenerative damage of neurosensorial acustic system, such as Ménière's disease [15]. Ménière's disease (MD) is characterized by the triad of fluctuating hearing loss, episodic vertigo and tinnitus, and by endolymphatic hydrops found on post-mortem examination. Increasing evidence suggests that oxidative stress is involved in the development of endolymphatic hydrops and that cellular damage and apoptotic cell death might contribute to the sensorineural hearing loss found in later stages of MD [16]. Consistent with this notion, studies are presently under way in our laboratory testing the conceivable possibility that mushrooms supplementation can reverse oxidative damage, thereby affecting the clinical course of Meniere's disease pathology. Thus, modulation of endogenous cellular defense mechanisms such as the vitagene network may open to new therapeutic approaches in diseases associated with tissue damage and cell death, such as in glaucomatous or Meniere neurodegeneration $[14,15]$. In this review, we specifically discuss the main neuroprotective and nutritional activities of Coriolus and Hericium mushrooms. Moreover, we will introduce the emerging role of hormesis and inflammasome as important components of neuroprotective network operating in redox-dependent brain cell stress responsive mechanisms. Our focus presently highlights the hypothesis linking oxidative stress and neurodegeneration to the $\mathrm{AD}$ pathogenesis, and indicate that stress responsive genes may represent an important target for novel cytoprotective strategies, as molecules inducing this defense mechanism, via nutritional and/or pharmacological approaches, can exploit the potential for antidegenerative therapeutic interventions [14].

\section{Neurohormesis}

At the core of adaptive responses at the cell and origin of biological organization is the concept of hormesis [17]. Hormesis is the expression of integrative adaptive responses that are manifest via a biphasic dose response with very specific quantitative response feature (i.e. maximum amplitude and width of the adaptive response) and induced by either a direct stimulatory response or as a modest overcompensation to a disruption of homeostasis 
[18]. Detailed assessments of pre- and post-conditioning, the adaptive response in radiation, the priming response, as commonly reported in microbial and plant models, and the so-called steeling effect widely reported in the clinical psychology area, all these phenomena act via hormetic mechanisms and comprise a broadly integrated and evolutionarily based adaptive system that has been highly conserved [19]. Such hormetic dose responses provide a quantitative description of the bounds of biological plasticity [20], and a measure of the extent to which adaptive processes may be upregulated, which is especially relevant to the comprehension of protective effects induced by plant and fungal species. The hormetic concept is particularly important since it provides reliable estimates of the upper limit for the induction of potential therapeutic responses and should play a key role in the design of experimental studies and clinical trials. Hormesis, expecially in vulnerable biological systems, such as the brain, is of relevant interest to the toxicological community for the dose-response model. Particularly, neurohormesis affects memory, learning and performance, as well as nutritional antioxidants and neurodegenerative responses mediated by oxidative stress in cellular models for various diseases such as $\mathrm{AD}[9,17]$. In fact, the presence of oxidative stress markers in the brains of patients with neurodegenerative diseases has been recognized which supports the rationale for neuroprotective nutritional interventions based on the action antioxidants and anti-inflammatory agents, such as polyphenols or mushrooms [7]. Neurohormesis can be applied to both polyphenol and nutritional mushroom molecular mechanisms of action. It has been, in fact, known that polyphenols and mushrooms activate the heat shock protein (Hsp) pathway, which plays a crucial role in the cellular stress response. With respect to this, for instance although there is no doubt about the protective effect of HO-1 against oxidative and nitrosative stress, on the contrary, excessive HO-1 upregulation may be toxic for cells. According to this principle, drugs, toxins and natural substances, administered at low doses can result in a positive response, while at higher concentrations promote prevalent toxic effects [17].

\section{Role of the inflammasome}

Oxidative stress is one of the main components of the pathogenesis of neurodegenerative diseases. The molecular mechanisms underlying oxidative stress include inflammation, mitochondrial dysfunction and apoptosis that culminates in neuronal death [21, 22]. Modulation of cellular stress pathways through the use of small redox active molecules represents a new approach to the study of neurologic and psychiatric pathologies [23]. In particular sulforaphane and hydroxytyrosol, as well as nutritional mushrooms are increasingly considered as possible candidates to regulate physiological pathways related with (1) cellular stress response and vitagene networks; (2) redox imbalance/oxidative stress, (3) mitochondrial function (4) immune response and anti-neuroinflammation (5) heat shock response control, and (6) synaptic dysfunction [24]. Excessive oxidative stress levels have been correlated to AD pathogenesis, increased protein carbonylation, nitration, cysteine-oxidation, lipid peroxidation, and DNA/ RNA oxidation, were revealed in brain and peripheral tissue samples from patients with $\mathrm{AD}$ by post-mortem studies. Increased oxidative stress can damage mitochondrial proteins. Of particular significance to the pathophysiology of main neurodegenerative disorders are findings of reduced levels of mRNA (and protein subunits) that are involved in the transfer of electrons in complex I of the electron transport chain (ETC), in AD patients. Decreased efficiency of the electron transfer process within complex I and complex IV, results in increased leakage and monoelectronic reduction of molecular oxygen to form the superoxide anion [25], with ensuing damage to proteins, lipids and DNA. As well, increasing evidence supports a role of immune activation as a prominent causative factor in the pathogenesis of a number of major neurologic and neuropsychiatric disorders [26]. Consistent with this notion, current studies have demonstrated that the inflammasome modulates neuroinflammatory processes at the initial stage, with a secondary cascade of events inclusive of oxidative stress, redox homeostasis disruption associated to mitochondrial dysfunction (Fig. 1) [27]. The inflammasome is a multiprotein complex that contains many copies of a receptor for pathogen- or damagederived molecular patterns (Pathogen Associated Molecular Patterns, PAMPs), pro-caspases-1, and an adaptor, apoptotic speck-containing protein with a caspase activation and recruitment domain (CARD) [ASC], which induces caspase-1 maturation [28]. Active caspase- 1 is responsible for rapid, lytic cell death (pyroptosis). Upon sensing PAMP or damage associated molecular pattern (DAMP), absent in melanoma 2 (AIM2) and/or PYD domains-containing protein 3 (NLRP3), inflammasomes activate caspase- 8 and caspase-1, respectively, leading to both pyroptotic and apoptotic cell death [29]. Mitochondria represent major sources of DAMPs capable of triggering neuroinflammatory responses, with resulting pyroptosis, apoptosis and autophagy [30]. AIM2 is a cytoplasmic sensor that recognizes and binds the doublestranded DNA (dsDNA) of microbial or host origin. Upon binding to DNA, AIM2 assembles inflammasome complex, which induces pyroptosis and proteolytic cleavage of the proinflammatory cytokines pro-IL-1 $\beta$ and pro-IL-18. A wrong recognition of cytoplasmic self-DNA by AIM2 provides to the development of autoimmune and inflammatory diseases, as well as neurodegenerative disorders [31].

A direct activation link between inflammation and the pathogenesis of Alzheimer's disease has been 


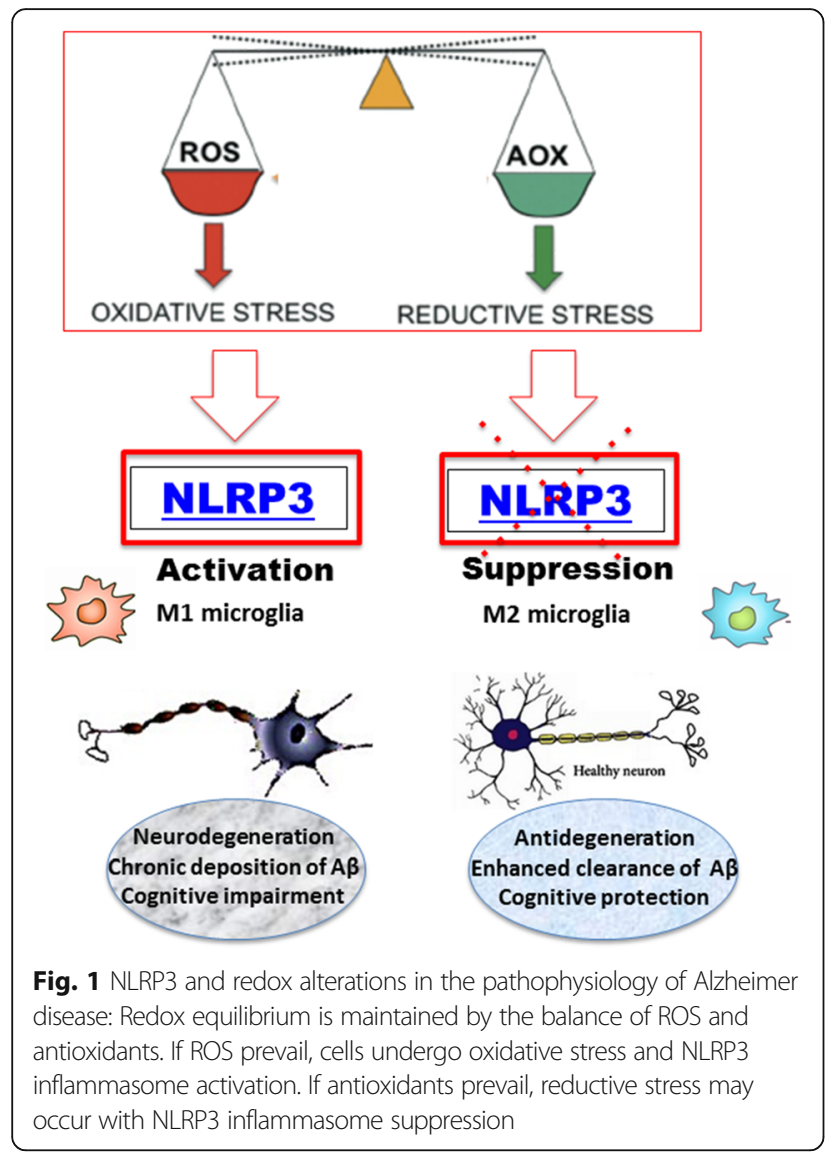

demonstrated by numerous in vitro and in vivo studies. in particular the inflammasome NLRP $£$ has been identified as a possible therapeutic target for the treatment of neurodegenerative diseases. This hypothesis was accredited by the observation of a deficiency of NLRP3 and caspase-1 in mice AA / PS1 (transgenic mice for chronic deposition of $\mathrm{Ab}$ ), in which $\mathrm{Ab}$ deposits were reduced, resulting in an increase in M2 microglia [32]. We have provided recent evidence of a neuroprotective action of the Hericium erinaceus (MRLs, UK) mushroom when administered orally to rat. In the brain of rats receiving oral administration of Hericium erinaceus, was measured maximum expression of LXA4, an anti-inflammatory compound, in cortex and hippocampus. LXA4 upregulation was related with an increased amount of proteins, such as thioredoxin, Hsp72 and heme oxygenase-1 involved in cellular stress response [4].

Plausibly, LXA4 signalling activation and stressresponsive vitagene proteins modulation could serve as a potential therapeutic target for AD-related inflammation and neurodegenerative damage. Our results indicate that nutritional supplementation with an opportune biomass preparation from a well characterized strain of Hericium erinaceus or Coriolus versicolor can induced critical proteins modulation involved in brain age-associated neurodegenerative diseases [33]. In view of the fact that in $\mathrm{AD}$ pathology, amyloid plaques accumulation (APs), composed of amyloid-beta peptide (A $\beta$ ) aggregates, and neurofibrillary tangles (NFTs) formation, composed of misfolded Tau proteins, are related with a deficit in those mechanisms participating in the induction of cytoprotective proteins ( $\mathrm{Hsps}$ ) or, more generally, cellular pathways of stress tolerance, it is conceivable to hypothesize that in these conditions, administration of Hericium erinaceus or Coriolus versicolor biomass, by enhancing the redox potential and inducing neuroprotection through neurohormetic mechanisms such as vitagenes upregulation, may promote resilience in damaged neurons, and hence resistance to proteotoxic insults and apoptotic neurodegeneration. Consistent with this concept, restoration of normal proteostasis is crucial for neuronal survival. Our research suggests new potential strategies based on the induction of vitagene defence system as a foundamental mechanism to promote proteome homeostasis and hence withstand pathological mechanisms associated to unhealthy aging of the brain associated to neurodegenerative diseases [4].

\section{Coriolus versicolor}

The medicinal properties of mushrooms have long been known to traditional medicine (Fig. 2a, b) [34, 35]. Antioxidant, anti-bacterial, and anti-viral properties have been attributed to mushrooms by controlled studies [36]. It has been shown that mushrooms are capable to stimulate the immune system of the host due to the high content of $\beta$ glucans, which activate many types of immune cells and stimulate cytokine responses [37-39]. Several of these polysaccharides are currently used in East countries in association to radio and chemotherapy [40]. In addition, Cordymin, a peptide with low molecular weight $(10,906 \mathrm{Da})$, with anti-inflammatory properties has been isolated from the medical mushroom Cordyceps sinensis [41] and from Cordyceps militaris [42]. This peptide significantly inhibited the polymorphonuclear cells infiltration and IR-induced up regulation of $\mathrm{C} 3$ protein produced in the brain, interleukin- $1 \beta$, and tumour necrosis factor- $\alpha$, which had a neuroprotective effect on the ischemic brain, due to the inhibition of inflammation [34].

Although the polysaccharides derived from mushrooms are hardly synthesizable molecules and the active molecules present in the mushrooms are still not well known, the Asian clinical practice employs preparations derived from mushrooms, including Agaricus campestris, Pleurotus ostreatus and Coriolus versicolor extracts [43]. Polysaccharides obtained from coriolus versicolor (Fig. 2a) are commercially among the best options. This mushroom is known for its medical applications, and is usually used to degrade organic contaminants such as 


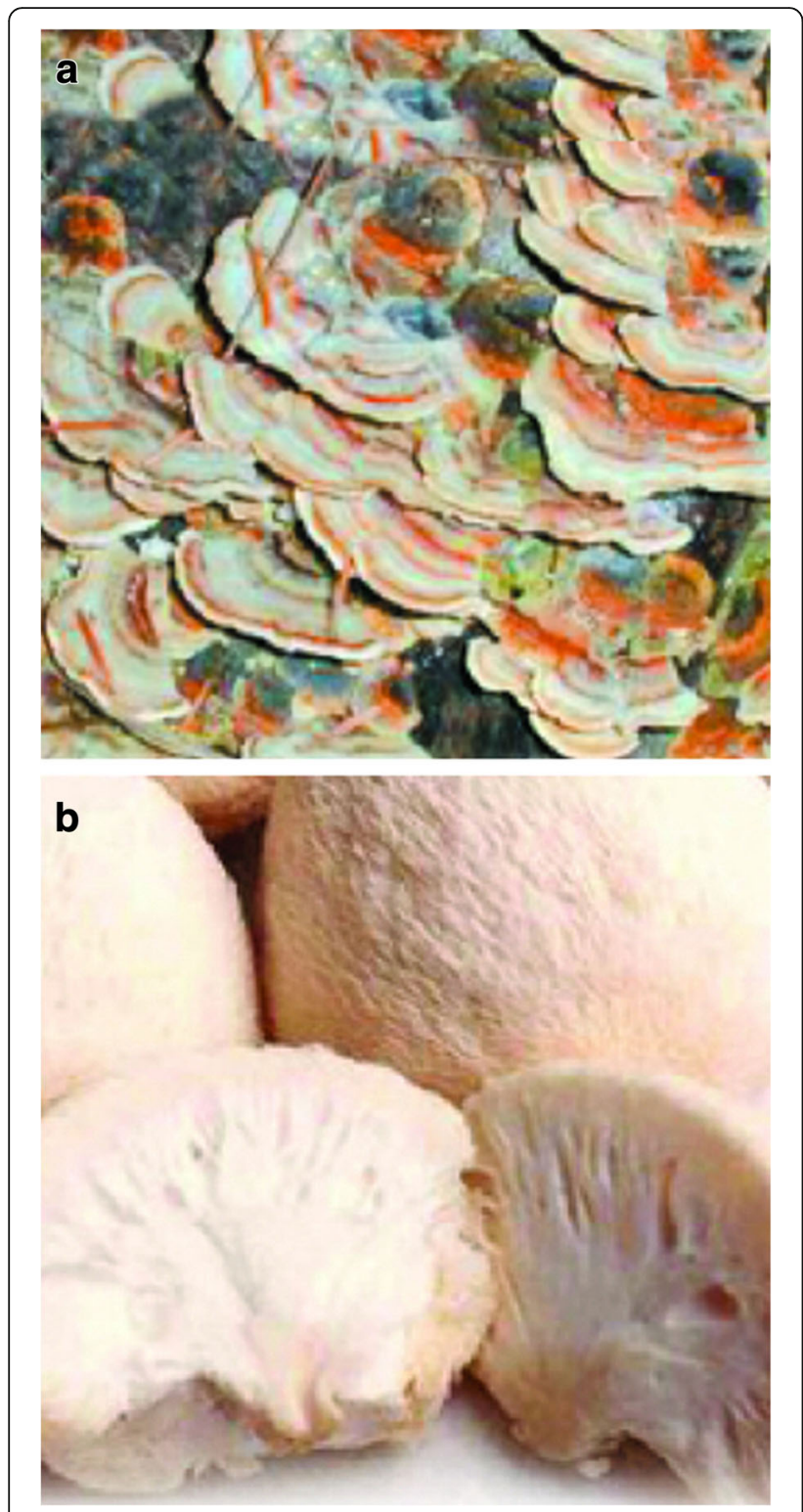

Fig. 2 Coriolus versicolor (a) and Hericium erinaceus (b) mushrooms

pentachlorophenol (PCP) [44]. Polysaccharide K or Kerstin (PSK) and polysaccharopeptide (PSP) are the main commercial preparations obtained by $\mathrm{C}$ versicolor mycelia [45]. These compounds stimulate the production of interleukin-6, interferons, immunoglobulin G, macrophages, and T-lymphocytes by enhancing the immune system against immunosuppressive effects of radiotherapy and chemotherapy. Polysaccharopeptides possess anticancer activity inhibiting the production of superoxide dismutase (SOD) and glutathione peroxidase [46]. Experimental animal and human studies have shown that oral administration of PSK and PSP controlled carcinomas [47]. It is reported that PSK produces apoptosis in HL-60 Human promyelocytic leukaemia cells through the activation of mitochondrial and caspase-dependent pathways [48] and overexpression of proapoptotic protein Bax [49]. Of particular interest are the polysaccaropeptides produced by Coriolus versicolor, which are used to supplement the chemotherapy and radiotherapy of cancer and infectious diseases. Chronic inflammation favours the progression of Alzheimer's disease (AD), however identification of mechanisms able of resolving the pro-inflammatory environment stimulating AD pathology remains an area of active investigation [50,51]. Taking into account this neurobiological rationale, our recent study, carried out to demonstrate a potential neuroprotective role of coriolus versicolor biomass preparation in neuroinflammatory pathogenesis associated with Alzheimer's disease in rat, has been undertaken to provide experimental evidence that biomass from Coriolus or Hericium regulate important redox sensitive pathways linked to cellular stress response and hence confer neuroprotection [3, 4, 52]. Lipoxin A4 (LXA4) derived from Coriolus is an endogenous eicosanoid capable to resolving inflammation process, acting as an endogenous "braking signal" in the inflammatory cascade. Treatment with the pro-resolving mediator aspirin-triggered lipoxin A4 (ATL), caused cognition improvement, reduced Ab levels, and enhanced phagocytic activity of microglia in Tg2576 transgenic AD mice [53]. Moreover, LXA4 levels declined with age, a finding even more evident in 3xTg-AD mice [54]. LXA4 action is regulated by the interaction with $G$ protein-coupled receptor. $\mathrm{N}$-formyl-peptide receptor 1 (FPRL1), also known as ALX (lipoxin A4 receptor) or CCR12, belongs to the formyl-peptide receptor (FPR)-related family of G protein-coupled receptors (GPCRs) that also includes FPR and FPRL2 [55, 56]. All factors able to increase Lipoxin A4 (LXA4) levels and consequently uptake of Ab by phagocytic cells are a hypothetical therapeutic target for $\mathrm{AD}$. Consistent with this notion, in $\mathrm{AD}$ pathology, the accumulation of amyloid plaques (APs), constituted of amyloid-beta peptide (Ab) aggregates, and neurofibrillary tangles (NFTs), constituted of misfolded Tau proteins, is related to a deficiency in the activation of cytoprotective proteins (Hsps) or, more generally, of cellular stress tolerance pathways [55]. In these conditions, it is demonstrated that the administration of Coriolus in the brain of rats causes the maximum induction of LXA4 in cortex and hippocampus. Notably, no significant modifications in I-Kappa-B-Alpha (IkBa), Nuclear Factor Kappa B (NFkB) and cyclooxygenase-2 (COX-2) brain levels were associated with Hsps induction [4]. Furthermore, LXA4 up-regulation is related with Nrf-2 regulated vitagenes, thus increasing the content of proteins involved in cellular oxidative stress response, such as thioredoxin, Hsp72 and heme oxygenase [52].Therefore the induction of vitagenes, could help 
vulnerable neurons resist proteotoxic insults and to reduce apoptotic neurodegeneration.

\section{Hericium erinaceus}

Hericium erinaceus (Fig. 2b) fruit bodies and mycelia contain an extraordinarily large quantity of structurally different bioactive and potentially bioactive components. The reported health-promoting properties of these compounds include anticarcinogenic, antibiotic, antidiabetic, antifatigue, antihypertensive, antihyperlipodemic, antisenescence, cardioprotective, nephroprotective, hepatoprotective, and neuroprotective properties and improvement of anxiety, depression and cognitive function [57]. The antioxidant activity of Hericium erinaceus has been observed in diabetic rat model in which the intra peritoneal (i.p.) administration of an aqueous extract of Hericium erinaceus (100 and $200 \mathrm{mg} / \mathrm{kg}$ body weight) resulted in a significant decrease in the serum glucose level, significant increase in the insulin level and attenuated serum lipid profiles (disorders) as compared to control rats. These findings were accompanied by increased activities in the antioxidative enzymes catalase (CAT), superoxide dismutase (SOD), and glutathione peroxidase (GSH-Px) and increased GSH (glutathione) and reduced malondialdehyde (MDA) levels in the liver, suggesting that the mechanism of the health-promoting effects seems to be the result of inhibition of ROS [58]. Endowed with different biological activities, Hericiumderived hericenones and erinacines, isolated from its fruiting body, stimulate nerve growth factor (NGF) synthesis in cultured astrocytes [59]. NGF influences basal forebrain cholinergic neurons modulating the activity of two enzymes, such as cholineacetyltransferase and acetyl cholinesterase. The first pathological events of Alzheimer's disease are the loss and dysfunction of cholinergic neurons. Hericium erinaceus administration improves cognitive dysfunction. Less, however, is known about the clinical relevance of Hericium erinaceus in regulating neurogenesis in the nervous system and its role in neurodegenerative disorders such as Alzheimer's disease and other types of dementia. Recently, basic and clinical studies have shown that Alzheimer's disease is closely associated with amyloid beta $(\mathrm{A} \beta)$-induced neuroinflammation, responsible for, the resident macrophages of the brain, and activated microglia may then promote neuronal injury through the release of proinflammatory and cytotoxic factors, exacerbating the course of the disease [60]. A new neuroprotective strategy, like an oral administration of a biomass Hericium biomass preparation given for 3 month, as done in our recent study [4] can represent a therapeutic target to minimize the deleterious effects related to oxidative burden, such as that occurring in brain aging and in neurodegenerative disorders. Treatment with Hericium erinaceus caused a significant increase of LXA4 production in most of the brain regions like cortex, hippocampus followed by substantia Nigra, striatum and cerebellum and in a modulated expression of cytoprotective proteins, such as Heme oxygenase 1 (HO-1), Heat Shock Protein 70 (Hsp70) and thioredoxin (TRX). These results are coherent with recent evidence obtained in mice, showing neuroprotection by Hericium erinaceus on A $\beta$ 25-35 peptide-induced cognitive dysfunction $[61,62]$.

\section{Conclusions}

Accumulating evidence has indicated that oxidative stress and excess reactive oxygen and nitrogen intermediates play an important role in the progression of many chronic inflammatory diseases, including cardiovascular diseases, diabetes, and neurodegenerative disorders [63]. Imbalance between ROS generation and antioxidant enzyme activities will cause lipid peroxidation, nuclear and mitochondrial DNA damage and protein oxidation, resulting in brain damage and amnesia [64]. A growing number of studies have demonstrated that dietary interventions regulate mitochondrial ROS production, detoxification and oxidative damage repair. Many (but not all) of these nutritional interventions are related with extension of lifespan, or protection against diseases related with age, in mammals. Emerging nutraceuticals are today showing promise as modulators of mitochondrial redox metabolism capable of eliciting beneficial outcomes. Mushrooms, known for their strong antioxidant properties, have attracted interest due to their potential in neuroprotection, antioxidant, and anti-inflammatory effects, as well as in proteome and mitochondrial homeostasis restoration as a basic mechanism to withstand mitochondrial dysfunction-associated neuroinflammatory disorders.

\section{Abbreviations}

AD: Alzheimer's disease; AIM2: Absent in melanoma 2; ALX: Lipoxin A4 receptor; APs: Amyloid plaques; ASC: Apoptotic speck-containing protein; ATL: Aspirin triggered lipoxin A4; AB: Amyloid-beta peptide; CAT: Catalase; CBF: Cerebral blood flow; COX-2: Cyclooxygenase-2; DAMP: Damage associated molecular pattern; ETC: Electron transport chain; FPRL1: N-formylpeptide receptor 1; GPCRs: G protein-coupled receptors; GSH: Glutathione; GSH-Px: Glutathione peroxidase; HO-1: Heme oxygenase 1; Hsp70: Heat shock protein 70; Hsp72: Heat shock protein 72; Hsps: Heat shock proteins; IkBa: I-Kappa-B-Alpha; LXA4: Lipoxin A4; MDA: Malondialdehyde; NFkB: Nuclear factor kappa B; NFTs: Neurofibrillary tangles; NGF: Nerve growth factor; NLRP3: PYD domains-containing protein 3; PCP: Pentachlorophenol; PD: Parkinson's disease; PSK: Polysaccharide K or Krestin; PSP: Polysaccharopeptide; RGC: Retinal ganglion cell; ROS: Radical oxygen species; SOD: Superoxide dismutase; TRX: Thioredoxin

\section{Acknowledgements \\ Not applicable}

\section{Funding}

Research activities in the area of dose response have been funded by the United States Air Force and ExxonMobil Foundation over a number of years. However, such funding support has not been used for the present manuscript (EJC)

Availability of data and materials

'Not applicable' in this section. 


\section{Authors' contributions}

"All authors had full access to the study and take responsibility for the integrity and the accuracy of the study concept and design. Drafting of the manuscript: ATS, MP, RDP, MS, RC, MTC, MLO, MT, MU, LM, EJC, SC, VC. ATS and MP equally contributed as first author. Critical revision of the manuscript for important intellectual content: VC, SC and EJC. Study supervision: VC and EJC." All authors read and approved the final manuscript.

\section{Ethics approval and consent to participate}

"Not applicable" in this section.

\section{Consent for publication}

Not applicable (the manuscript contains no individual person's data).

\section{Competing interests}

The authors declare that they have no competing interests.

\section{Publisher's Note}

Springer Nature remains neutral with regard to jurisdictional claims in published maps and institutional affiliations.

\section{Author details \\ 'Department of Biomedical and Biotechnological Sciences, School of Medicine, University of Catania, Via Santa Sofia 97, 95123 Catania, Italy. ${ }^{2}$ Department of Chemical, Biological, Pharmaceutical and Environmental Sciences University of Messina, Messina, Italy. ${ }^{3}$ Department of Medical and Surgery Sciences and Advanced Technology, University of Catania, Catania, Italy. "Spinal Unit, Emergency Hospital "Cannizzaro", Catania, Italy. \\ ${ }^{5}$ Environmental Health Sciences Division, School of Public Health, University of Massachusetts, Amherst, MA, USA.}

\section{Received: 4 October 2017 Accepted: 28 December 2017}

\section{Published online: 14 February 2018}

\section{References}

1. Ziegler-Graham K, Brookmeyer R, Johnson E, Arrighi HM. Worldwide variation in the doubling time of Alzheimer's disease incidence rates. Alzheimer Dement. 2008;4(5):316-23.

2. Blennow K, Zetterberg H. Pinpointing plaques with PIB. Nat Med. 2006;12(7):753-4.

3. Calabrese V, Cornelius C, Dinkova-Kostova AT, Calabrese EJ, Mattson MP. Cellular stress responses, the hormesis paradigm, and vitagenes: novel targets for therapeutic intervention in neurodegenerative disorders. Antioxid Redox Signal. 2010;13:1763-811.

4. Trovato Salinaro A, Siracusa R, Di Paola R, Scuto M, Ontario ML, Bua O, et al. Redox modulation of cellular stress response and lipoxin A4 expression by Hericium erinaceus in rat brain: relevance to Alzheimer's disease pathogenesis. Immun Ageing. 2016;13:23.

5. Kirstein J, Morito D, Kakihana T, Sugihara M, Minnen A, Hipp MS, et al. Proteotoxic stress and ageing triggers the loss of redox homeostasis across cellular compartments. EMBO J. 2015;34:2334-49.

6. Calabrese V, Dattilo S, Petralia A, Parenti R, Pennisi M, Koverech G, et al. Analytical approaches to the diagnosis and treatment of aging and aging-related disease: redox status and proteomics. Free Radic Res. 2015;49:511-24

7. Calabrese V, Giordano J, Signorile A, Laura Ontario M, Castorina S, De Pasquale C, et al. Major pathogenic mechanisms in vascular dementia: roles of cellular stress response and hormesis in neuroprotection. J. Neurosci Res. 2016;94: 1588-603.

8. Yang F, Xie J, Wang W, Xie Y, Sun H, Jin Y, et al. Regional arterial infusion with lipoxin A4 attenuates experimental severe acute pancreatitis. PLoS One. 2014;9:108525.

9. Calabrese V, Giordano J, Ruggieri M, Berritta D, Trovato A, Ontario ML, et al. Hormesis, cellular stress response, and redox homeostasis in autism spectrum disorders. J Neurosci Res. 2016;94:1488-98.

10. Hawkins KE, DeMars KM, Singh J, Yang C, Cho HS, Frankowski JC, et al. Neurovascular protection by post-ischemic intravenous injections of the lipoxin A4 receptor agonist, BML-111, in a rat model of ischemic stroke. J Neurochem. 2014;129:130-42.

11. Cesareo M, Martucci A, Ciuffoletti E, Mancino R, Cerulli A, Sorge RP, et al. Association between Alzheimer's disease and glaucoma: a study based on
Heidelberg retinal tomography and frequency doubling technology Perimetry. Front Neurosci. 2015;9:479.

12. Tham YC, Li X, Wong TY, Quigley HA, Aung T, Cheng CY. Global prevalence of glaucoma and projections of glaucoma burden through 2040: a systematic review and meta-analysis. Ophthalmology. 2014;121:2081-90.

13. Carelli V, La Morgia C, Valentino ML, Barboni P, Ross-Cisneros FN, Sadun AA. Retinal ganglion cell neurodegeneration in mitochondrial inherited disorders. Biochim Biophys Acta. 2009:518-28.

14. Trovato Salinaro A, Cornelius C, Koverech G, Koverech A, Scuto M, Lodato F, Fronte V, Muccilli V, Reibaldi M, Longo A, Uva MG, Calabrese V. Cellular stress response, redox status, and vitagenes in glaucoma: a systemic oxidant disorder linked to Alzheimer's disease. Front Pharmacol. 2014;5:129. https:// doi.org/10.3389/fphar.2014.00129.

15. Calabrese V, Cornelius C, Maiolino L, Luca M, Chiaramonte R, Toscano MA, Serra A. Oxidative stress, redox homeostasis and cellular stress response in Ménière's disease: role of vitagenes. Neurochem Res. 2010;35:2208-17.

16. Requena T, Cabrera S, Martín-Sierra C, Price SD, Lysakowski A, LopezEscamez JA. Identification of two novel mutations in FAM136A and DTNA genes in autosomal-dominant familial Meniere's disease. Hum Mol Genet. 2015;24:1119-26.

17. Calabrese EJ, Mattson MP. How does hormesis impact biology, toxicology, and medicine? NPJ Aging Mech Dis. 2017;3:13.

18. Calabrese EJ, Baldwin LA. The frequency of U-shaped dose responses in the toxicological literature. Toxicol Sci. 2001;62:330-8.

19. Calabrese EJ, Bachmann KA, Bailer AJ, Bolger PM, Borak J, Cai L, et al. Biological stress response terminology: integrating the concepts of adaptive response and preconditioning stress within a hormetic dose-response framework. Toxicol App Pharmacol. 2007;222:122-8.

20. Calabrese EJ, Mattson MP. Hormesis provides a generalized quantitative estimate of biological plasticity. J Cell Commun Sign. 2011;5:25-38.

21. Lim JH, Gerhart-Hines Z, Dominy JE, Lee Y, Kim S, Tabata M, et al. Oleic acid stimulates complete oxidation of fatty acids through protein Kinase A-dependent activation of SIRT1-PGC1 alpha complex. J Biol Chem. 2013;288:7117-26.

22. Guest J, Garg M, Bilgin A, Grant R. Relationship between central and peripheral fatty acids in humans. Lipids Health Dis. 2013:12:30-7.

23. Liu Z, Zhou T, Ziegler AC, Dimitrion P, Zuo L. Oxidative stress in neurodegenerative diseases: from molecular mechanisms to clinical applications. Oxidative Med Cell Longev. 2017;23:45-55.

24. Patel S, Goyal A. Recent developments in mushrooms as anti-cancer therapeutics: a review. 3. Biotech. 2012;2:1-15.

25. Talalay P, Zimmerman AW. Reply to Scahill: behavioral outcome measures in autism. Proc Natl Acad Sci U S A. 2015;112:E350-E.

26. Hroudova J, Singh N, Fisar Z. Mitochondrial dysfunctions in neurodegenerative diseases: relevance to Alzheimer's disease. Biomed Res Int. 2014;56:89-99.

27. Morris $G$, Berk M. The many roads to mitochondrial dysfunction in neuroimmune and neuropsychiatric disorders. BMC Med. 2015;13:34-47.

28. Kim YK, Shin JS, Nahm MH. NOD-like receptors in infection, immunity, and diseases. Yonsei Med J. 2016:57:5-14.

29. Davis BK, Wen HT, Ting JPY. The Inflammasome NLRs in immunity, inflammation, and associated diseases. Annu Rev Immunol. 2011;29:707-35.

30. Aachoui Y, Sagulenko V, Miao EA, Stacey KJ. Inflammasome-mediated pyroptotic and apoptotic cell death, and defense against infection. Curr Opin Microbiol. 2013;16:319-26.

31. Bosch ME, Kielian T. Neuroinflammatory paradigms in lysosomal storage diseases. Front Neurosci-Switz. 2015;9:33-46.

32. Gadani SP, Walsh JT, Lukens JR, Kipnis J. Dealing with danger in the CNS: the response of the immune system to injury. Neuron. 2015;87:47-62.

33. Heneka MT. Inflammasome activation and innate immunity in Alzheimer's disease. Brain Pathol. 2017;27:220-2

34. Cornelius C, Graziano A, Calabrese EJ, Calabrese V. Hormesis and vitagenes in aging and longevity: mitochondrial control and hormonal regulation. Horm Mol Biol Clin Investig. 2013;16:73-89.

35. Wang D, Calabrese EJ, Lian B, Lin Z, Calabrese V. Hormesis as a mechanistic approach to understanding herbal treatments in traditional Chinese medicine. Pharmacol Ther. 2017. https://doi.org/10.1016/j.pharmthera.2017. 10.013. Nov 8. pii: S0163-7258(17)30263-2.

36. Elsayed EA, El Enshasy H, Wadaan MA, Aziz R. Mushrooms: a potential natural source of anti-inflammatory compounds for medical applications. Mediat Inflamm. 2014;2014:835-41. 
37. Paterson RR, Lima N. Biomedical effects of mushrooms with emphasis on pure compounds. Biom J. 2014;37:357-68.

38. Komura DL, Ruthes AC, Carbonero ER, Gorin PA, lacomini M. Water-soluble polysaccharides from Pleurotus ostreatus var. florida mycelial biomass. Int Biol Macromol. 2014;70:354-9.

39. Wasser SP. Medicinal mushroom science: current perspectives, advances, evidences, and challenges. Biom J. 2014;37:345-56.

40. Lindequist U, Kim HW, Tiralongo E, Van Griensven L. Medicinal mushrooms. Evid Based Complement Alternat Med. 2014;12:1-2.

41. Xu T, Beelman RB, Lambert JD. The cancer preventive effects of edible mushrooms. Anti Cancer Agents Med Chem. 2012;12:1255-63.

42. Wang J, Liu YM, Cao W, Yao KW, Liu ZQ, Guo JY. Anti-inflammation and antioxidant effect of Cordymin, a peptide purified from the medicinal mushroom Cordyceps sinensis, in middle cerebral artery occlusion-induced focal cerebral ischemia in rats. Metab Brain Dis. 2012;27:159-65.

43. Wong JH, Ng TB, Wang H, Sze SC, Zhang KY, Li Q, et al. Cordymin, an antifungal peptide from the medicinal fungus Cordyceps militaris. Phytomedicine. 2011;18:387-92

44. Cui J, Goh KK, Archer R, Singh H. Characterisation and bioactivity of proteinbound polysaccharides from submerged-culture fermentation of Coriolus versicolor Wr-74 and ATCC-20545 strains. J Ind Microbiol. 2007;34:393-402.

45. Trovato SA, Pennisi M, Crupi R, Di Paola R, Alario A, Modafferi S, Di Rosa G, Fernandes T, Signorile A, Maiolino L, Cuzzocrea S, Calabrese V. Neuroinflammation and mitochondrial dysfunction in the pathogenesis of Alzheimer's disease: modulation by Coriolus Versicolor (Yun-Zhi) nutritional mushroom. J Neurol Neuromed. 2017;2:19-28.

46. Cui J, Chisti Y. Polysaccharopeptides of Coriolus versicolor: physiological activity, uses, and production. Biotechnol Adv. 2003;21:109-22.

47. $\mathrm{Ng} \mathrm{TB}$. A review of research on the protein-bound polysaccharide (polysaccharopeptide, PSP) from the mushroom Coriolus versicolor (Basidiomycetes: Polyporaceae). Gen Pharmacol. 1998;30:1-4.

48. Hirahara N, Edamatsu T, Fujieda A, Fujioka M, Wada T, Tajima Y. Proteinbound polysaccharide-K induces apoptosis via mitochondria and p38 mitogen-activated protein kinase-dependent pathways in HL-60 promyelomonocytic leukemia cells. Oncol Rep. 2013;30:99-104.

49. Ho CY, Kim CF, Leung KN, Fung KP, Tse TF, Chan H, et al. Coriolus versicolor (Yunzhi) extract attenuates growth of human leukemia xenografts and induces apoptosis through the mitochondrial pathway. Oncol Rep. 2006;16:609-16.

50. Figueiredo-Pereira ME, Rockwell P, Schmidt-Glenewinkel T, Serrano P. Neuroinflammation and $\mathrm{J} 2$ prostaglandins: linking impairment of the ubiquitin-proteasome pathway and mitochondria to neurodegeneration. Front Mol Neurosci. 2015;7

51. Joshi YB, Pratico D. The 5-lipoxygenase pathway: oxidative and inflammatory contributions to the Alzheimer's disease phenotype. Front Cell Neurosci. 2015; 8:436. https://doi.org/10.3389/fncel.2014.00436.

52. Trovato Salinaro A, Siracusa R, Di Paola R, Scuto M, Fronte V, Koverech G, Luca M, Serra A, Toscano MA, Petralia A, Cuzzocrea S, Calabrese V. Redox modulation of cellular stress response and lipoxin A4 expression by Coriolus versicolor in rat brain: relevance to Alzheimer's disease pathogenesis. Neurotoxicology. 2016;53:350-8.

53. Dunn HC, Ager RR, Baglietto-Vargas D, Cheng D, Kitazawa M, Cribbs DH, et al. Restoration of lipoxin A4 signaling reduces Alzheimer's disease-like pathology in the 3xTg-AD mouse model. J Alzheimers Dis. 2015:43:893-903.

54. Gangemi S, Pescara L, D'Urbano E, Basile G, Nicita-Mauro V, Davi G, et al. Aging is characterized by a profound reduction in anti-inflammatory lipoxin A4 levels. Exp Gerontol. 2005;40:612-4.

55. Le Y, Murphy PM, Wang JM. Formyl-peptide receptors revisited. Trends Immunol. 2002;23(11):541-8.

56. Chiang N, Serhan CN, Dahlen SE, Drazen JM, Hay DW, Rovati GE, et al. The lipoxin receptor ALX: potent ligand-specific and stereoselective actions in vivo. Pharm Rev. 2006;58:463-87.

57. Friedman M. Chemistry, nutrition, and health-promoting properties of Hericium erinaceus (Lion's mane) mushroom fruiting bodies and mycelia and their bioactive compounds. J Agric Food Chem. 2015;63:7108-23.

58. Liang B, Guo ZD, Xie F, Zhao AN. Antihyperglycemic and antihyperlipidemic activities of aqueous extract of Hericium ein experimental diabetic rats. BMC Complement Altern Med. 2013;13:253. https://doi.org/10.1186/1472-688213-253.

59. Lai CL, Lin RT, Liou LM, Liu CK. The role of event-related potentials in cognitive decline in Alzheimer's disease. Clin Neurophysiol. 2010;121:194-9.
60. Mori K, Inatomi S, Ouchi K, Azumi Y, Tuchida T. Improving effects of the mushroom Yamabushitake (Hericium erinaceus) on mild cognitive impairment: a double-blind placebo-controlled clinical trial. Phytother Res. 2009:23:367-72.

61. Wu J, Wang AT, Min Z, Xiong YJ, Yan QY, Zhang JP, et al. Lipoxin A(4) inhibits the production of proinflammatory cytokines induced by beta-amyloid in vitro and in vivo. Biochem Biophys Res Commun. 2011;408:382-7.

62. McGeer PL, McGeer EG. Innate immunity, local inflammation, and degenerative disease. Sci Aging Knowl Environ. 2002;3:23-32.

63. Nakamura T, Cho DH, Lipton SA. Redox regulation of protein misfolding, mitochondrial dysfunction, synaptic damage, and cell death in neurodegenerative diseases. Exp Neurol. 2012;238:12-21.

64. Biasibetti R, Tramontina AC, Costa AP, Dutra MF, Quincozes-Santos A, Nardin $P$, et al. Green tea (-)epigallocatechin-3-gallate reverses oxidative stress and reduces acetylcholinesterase activity in a streptozotocin-induced model of dementia. Behav Brain Res. 2013;236:186-93.

\section{Submit your next manuscript to BioMed Central and we will help you at every step:}

- We accept pre-submission inquiries

- Our selector tool helps you to find the most relevant journal

- We provide round the clock customer support

- Convenient online submission

- Thorough peer review

- Inclusion in PubMed and all major indexing services

- Maximum visibility for your research

Submit your manuscript at www.biomedcentral.com/submit
) Biomed Central 